\title{
Investigations of Silica Alcogel Aging Using Coherent Light
}

\author{
Arlon J. Hunt,* Michael R. Ayers, \\ E.O. Lawrence Berkeley National Laboratory, Berkeley, CA, 94720, USA
}

\begin{abstract}
Light scattering methods have previously been used to monitor the formation of gels. In this report we present new light scattering techniques to study the properties of silica alcogels during the aging process. Monitoring one particular polarization transformation of scattered light with time reveals a clear increase in internal strain in standing alcogels with time. The stress birefringence coefficient of an acid-catalyzed $\mathrm{SiO}_{2}$ gel was found to be $3.4 \times 10^{12}$ Brewsters. Additionally, the evolution of the stiffness of alcogels was investigated using laser speckle methods. Specifically, image analysis of specklegrams obtained during multi-frequency acoustic excitation of aging gels was used to non-destructively measure the hardening of alcogels. For an acidcatalyzed gel with a theoretical density of $\sim 0.05 \mathrm{~g} / \mathrm{cm}^{3} \mathrm{SiO}_{2}$, the rate of hardening is found to be greatest between gelation and $2 \mathrm{X}$ the gel time, and drops considerably thereafter. The Young's modulus of the gel can be monitored over time with this method and was found to range from $6.2 \times 10^{3} \mathrm{~N} / \mathrm{m}^{2}$ after 6 hours to $2.2 \times 10^{5} \mathrm{~N} / \mathrm{m}^{2}$ after 24 hours for acid-catalyzed silica gels.

PACS Codes: 42.25.Fx; 81.20.Fw; 82.70.Gg

${ }^{*}$ Corresponding author. Tel: +1-510-486-5370; fax: +1-510-486-7303; e-mail: ajhunt@1bl.gov
\end{abstract}




\section{Introduction}

Light scattering methods have been used extensively to study the dynamic behavior, pore structure, and other properties of silica sols and gels [1-5]. These methods have also been employed for gels that have been supercritically dried to aerogels [6]. However, light scattering methods are not limited to the study of sols prior to gelation, or the final solid product, but may be used to observe other processes, such as gel aging, as well. We present here two light scattering techniques that have been used for the first time to monitor the aging and hardening of silica gels prior to drying.

The aging process plays a critical role in determining the final mechanical, textural, and optical properties of an aerogel produced by sol-gel methods. These factors greatly affect the potential use of silica aerogels in commercial applications. Therefore detailed understanding of the aging process is desirable to improve the material quality of silica aerogels.

The polarization of light scattered from a gel (or aerogel) resulting from a given input polarization can be used to probe the mechanical properties of the gel. As the gel matures internal stresses build up that arise from adherence to the container, non-uniform solvent escape, syneresis, and external forces. These stresses give rise to anisotropic changes in the gel that induce different propagation velocities for light polarized parallel and perpendicular to the stress. These differences are sensitively probed by the polarization of light propagating in the gel. We quantify these changes using the polarization scatterometer that measures the retardance or degree to which linearly polarized light is transformed to circularly polarized light [7]. Specifically we measure the $\mathrm{S}_{34}$ Mueller matrix element as a function of scattering angle. The instrument utilizes 
polarization modulation with synchronous detection and can measure all of the polarization changes induced by the gel.

In an earlier paper [8], we reported the use of laser speckle contrast methods to observe the effect of catalyst concentration on the aggregation behavior of silica sols. Here, we extend this method by measuring acoustically coupled vibration of silica gels to monitor their aging behavior. Observation of the speckle contrast produced when a laser light source scatters off a vibrating medium has been used for some time to quantitatively measure the vibrational modes of the medium [9]. Vibration of the medium produces a corresponding vibration in the specklegram, which, if the period of the vibration is shorter than the image exposure time, produces a specklegram with reduced contrast. In the case of silica gels, the modulus of the medium is changing with time as the gel hardens, and therefore the frequency of optimum coupling of the external acoustic energy to the gel the changes with time. Monitoring the contrast of a specklegram produced from an aging silica gel that is exposed to acoustic energies of various frequencies can then be used to observe the aging time required for the gel to harden to a particular value. These results can be compared to the data from polarization measurements to provide a unified view of silica gel aging.

\section{Experimental procedures}

The silica gels used in this study were all derived from tetraethoxysilane (TEOS, reagent grade, EM Science). Acid-catalyzed gels used the following reagent amounts; $18 \mathrm{~mL}$ TEOS, $76 \mathrm{~mL}$ ethanol, $6.6 \mathrm{~mL} \mathrm{H}_{2} \mathrm{O}, 0.30 \mathrm{~mL} 52 \% \mathrm{HF}$. Base catalyzed gels used the same amounts of reagents, except for $0.036 \mathrm{~mL} 0.5 \mathrm{M} \mathrm{NH}_{4} \mathrm{~F}$ and $0.0 .048 \mathrm{~mL} 30 \%$ 
$\mathrm{NH}_{3}$, which were used in place of HF. In all cases, two solutions were prepared, one containing TEOS and half of the ethanol, and the other the remaining reagents. These were quickly mixed and filtered through $1 \mu \mathrm{m}$ PTFE filters directly into the vessel used for the scattering experiment.

For the polarization measurements, the gels were poured into a clear container fabricated from glass tubing with a diameter of $5 \mathrm{~cm}$. This arrangement provided a cylindrical gel with one end accessible for applying additional stress for calibration. After pouring, the gels were sealed and placed in the scatterometer and the $\mathrm{S}_{34}$ matrix element was monitored as a function of time. The scatterometer measures the $\mathrm{S}_{34}$ at 10degree scattering angle increments from 30 to 150 degrees. The retardance, $\Gamma$, is determined from $\mathrm{S}_{34}$ by the relationship $\Gamma=\pi \mathrm{S}_{34} / 2$. To calibrate the stress measurement a piston in contact with top of the gel was loaded with various weights and the corresponding retardance was measured.

Laser speckle contrast measurements employed an apparatus that has been previously described [8] with the addition of a controllable signal generator (Agilent 33120 A) whose output was sent to speakers placed $\sim 15 \mathrm{~cm}$ from the gel sample. The software which controls image acquisition and analysis (XCAP v. 1.0, Epix, Inc.) also controlled the signal generator via an RS-232 connection. The following procedure was used to monitor the aging of gels: the image contrast was measured for 10 images taken sequentially, averaged, and recorded along with the time of measurement with no external sound; a second set of 10 images were obtained while the system produced a frequency of $50 \mathrm{~Hz}$ and the average contrast and time were recorded in a separate file; additional measurement were similarly made at 150, 175, 200, 250, and $300 \mathrm{~Hz}$. The 
period required to acquire and process the images at these 7 frequencies was $\sim 80 \mathrm{sec}$. The system then repeated this series of measurements continuously over 72 hours, and, in this way, produced seven interlaced sets of data corresponding to each chosen frequency. Samples were contained in 20 -mL glass scintillation vials that were filled completely with sol touching the cap.

\section{Results}

Plots of the $\mathrm{S}_{34}$ matrix element versus angle for acid-catalyzed silica gels appear in Fig. 1. Data taken at 6 hours, 120 hours, and 120 hours with an applied force of 6.4 psi are shown. Commonly, strain birefringence is observed by high modulations at both high and low angles (with opposite signs), and near-zero modulation at $90^{\circ}$. This is seen here, with modulations at $30^{\circ}$ of near zero after 6 hours, $\sim 10 \%$ after 120 hours, and $\sim 20 \%$ with external pressure. . The stress birefringence coefficient of an acid-catalyzed $\mathrm{SiO}_{2}$ gel was calculated from this data to be $1.3 \times 10^{12}$ Brewsters or roughly similar to that for fused silica of $3.4 \times 10^{12}$ Brewsters. Time dependent syneresis, a slightly lower fundamental particle density (commonly $2.0-2.1 \mathrm{~g} / \mathrm{cm}^{3}$, for sol-gel silica), and solvent effects on the bonding of the primary silica particles are probably responsible for the difference in the values.

Figure 2 gives a stacked plot of speckle contrast vs. time for several cases of applied external acoustic energy. The gel time is marked with a dashed vertical line. The portion of the plots to the left of the line shows the broad peak characteristic of the sol-togel transition previously described [8]. To the right of the line is the region associated with the aging of the gel. In each case, three main peaks can be seen (marked by dashes 
on the plots) where the gel couples to the external vibrations. The positions of these peaks at each frequency are given in Table 1. Careful inspection of the plots shows additional smaller peaks coincident with those at integral multiples of frequency that correspond to coupling with higher harmonics.

The positions of the three main peaks shift to longer times as the frequency of the external vibrations is increased, and the spacing between the peaks increases as well. This is shown in Fig. 3, which plots the normalized time between the first and third peak vs. the position of the first. The plot shows that the rate of gel hardening is greatest immediately after the get point, and decreases substantially thereafter.

The Young's modulus for a gel can be determined from these data by using:

$$
E=f^{2} \lambda^{2} \rho
$$

where, $\mathrm{E}$ is the Young's modulus, $f$ is the frequency, $\lambda$ is the wavelength of the fundamental vibration, and $\rho$ is the density of the medium. This corresponds to the wave equation expected for longitudinal vibrations in a closed cylinder. Using a density of $0.82 \mathrm{~g} / \mathrm{cm}^{3}$, and a wavelength (determined by the size of the container) of $0.055 \mathrm{~m}$, and the frequencies from Table 1, values of Young's modulus ranging from $6.2 \times 10^{3}$ to $2.2 \mathrm{x}$ $10^{5} \mathrm{~N} / \mathrm{m}^{2}$ can be calculated. Assuming that the first major peak in each plot from Fig. 2 corresponds to the fundamental for a longitudinal vibrational mode, a plot of Young's modulus vs. time can be produced (Fig. 4).

\section{Discussion}

The observation of silica gel aging provides important internal information on the internal stress within the gel. These stresses play a major role in determining the potential 
utility of subsequent aerogels, as undesirable effects such as spontaneous cracks, and shape distortions may result. For acid catalyzed gels, we detected a stress of $\sim 44 \mathrm{kPa}$ in a $100 \mathrm{~mL}$ cylindrical gel after 4 days aging. However, this was observed while the gel was still contained in its original glass mold. Removing gels from their molds before the aging process, or using molds with unreactive surfaces, may reduce the formation of internal stresses.

The measurement of Young's modulus by laser speckle contrast imaging with acoustic coupling provides a convenient method for monitoring the hardening of gels during the aging process. We have shown that while the rate of aging slows dramatically after $\sim 2 \mathrm{x}$ the gel time, acid-catalyzed silica gels continue to harden for days or weeks beyond the gel point. The magnitude of the gel modulus is also easily measured and we have shown that for acid-catalyzed gels an increase on the order of $10^{3}$ to up to $10^{6} \mathrm{~N} / \mathrm{m}^{2}$ occurs over several days or weeks. This is in agreement with earlier results obtained for silica aerogels of various densities [10].

Interestingly, this method is, as yet, not as applicable to base-catalyzed gels. While these show similar speckle contrast evolution to that of acid-catalyzed gels in the early stages of gelation, their post-gelation behavior is quite different. In contrast to the sharp, well-defined peaks seen in the acid-catalyzed gels, basic gels show very broad peaks with multiple shoulders. These are seen at several acoustic frequencies, and may last for several hours. This suggests a very different hardening rate, and a fundamentally different set of mechanical properties is involved in the basic system. 


\section{Conclusions}

We have developed two new optical methods for monitoring the aging process of gels. Polarization-modulated light scattering provides information on both internal and external stresses present in aging gels. Laser speckle contrast imaging with acoustic coupling reveals the dynamic evolution of the mechanical properties of aging gels, specifically, the rate of evolution and magnitude of the gel modulus. Both methods have the advantage that they can be performed without disturbing the gels or removing them from their containers. These techniques have been used to emphasize the importance of long term aging of gels and the differences in the acid- and base-catalyzed silica systems. 


\section{References}

[1] J.E. Martin, K.D. Keefer, Phys. Rev. A, 34 (1986), 4988.

[2] A.H. Boonstra, T.P.M. Meeuwsen, J.M.E. Baken, G.V.A. Aben. , J. Non-Cryst. Solids, 109 (1989), 153.

[3] A.J. Hunt, J Non-Cryst. Solids, 225 (1998), 303.

[4] A.J. Hunt, M.R. Ayers, J Non-Cryst. Solids, 225 (1998), 325.

[5] Y. Xu, Y. Koga, Clays and Clay Min., 46 (1998), 161.

[6] A.J. Hunt, P. Berdahl, Mat. Res. Soc. Symp. Proc., 32 (1984), 275.

[7] M.S. Quinby-Hunt, P. G. Hull, A.J. Hunt, in M.L. Mischenko, J.W. Hovenier, and L.D. Travis (Eds.) "Light Scattering by Non- Spherical Particles: Theory, Measurements, and Geophysical Applications." Chapter 18, 2000, Academic Press, San Diego, London.

[8] M.R. Ayers, A.J. Hunt, J Non-Cryst. Solids, submitted for publication.

[9] B. Eliasson, F.M. Mottier, J. Opt. Soc. Am.., 61 (1971), 559.

[10] M. Gronauer, A. Kadur, J. Fricke, in: J. Fricke (Ed.) Springer Proc, Phys. 6, 1986 p. 167 Springer-Verlag, Berlin, New York. 


\section{Figure Captions}

Figure 1. $\mathrm{S}_{34}$ matrix element versus angle for acid-catalyzed TEOS gel; $\boldsymbol{\square}=$ after 6 hours, $\diamond=$ after 120 hours, $\bullet=$ after 120 hours with 6.4 psi external pressure.

Figure 2. Stacked plot of speckle contrast vs. time for an acid-catalyzed TEOS gel with various external acoustic frequencies applied. $\mathrm{T}_{\text {gel }}$ is marked by the dashed vertical line. Three main peaks indicating vibrational coupling to the external acoustic energy are seen in each plot and are marked with solid dashes.

Figure 3. Plot of normalized peak spacing, $T_{1} /\left(T_{3}-T_{1}\right)$, vs time of Peak $1\left(T_{1}\right)$ from speckle contrast plots for an acid-catalyzed teos gel. $\mathrm{T}_{\text {gel }}$ is marked by the dashed vertical line.

Figure 4. Plot of Young's Modulus vs. Time derived from acoustic measurements for an acid-catalyzed silica gel. $\mathrm{T}_{\text {gel }}$ is marked by the dashed vertical line. 


\section{Table 1}

Peak positions (time after mixing sol) observed with various external vibrations.

\begin{tabular}{llll}
\hline Frequency $(\mathrm{Hz})$ & $\begin{array}{l}\text { Peak 1 } \\
(\mathrm{min})\end{array}$ & $\begin{array}{l}\text { Peak 2 } \\
(\mathrm{min})\end{array}$ & $\begin{array}{l}\text { Peak 3 } \\
(\mathrm{min})\end{array}$ \\
\hline 50 & 376 & 395 & 402 \\
150 & 490 & 539 & 557 \\
175 & 571 & 650 & 678 \\
200 & 681 & 800 & 850 \\
250 & 1023 & 1283 & 1379 \\
300 & 1564 & 2087 & 2334
\end{tabular}

